\title{
Ultraschallwandler mit piezoelektrischen Funktionspolymeren für die Untersuchung des Trocknungs- bzw. Härtungsverhaltens von Lacken und Klebstoffen
}

\author{
Michael Wegener ${ }^{1}$, Harald Oehler ${ }^{2}$, Dirk Lellinger ${ }^{3}$ und Ingo Alig ${ }^{4}$ \\ ${ }^{1}$ Fraunhofer IAP, Geiselbergstrasse 69, 14469 Potsdam-Golm, 0331-5681209, \\ michael.wegener@iap.fraunhofer.de \\ ${ }^{2}$ Deutsches Kunststoff-Institut, Schloßgartenstrasse 6, 64289 Darmstadt, 06151-162406, \\ hoehler@dki.tu-darmstadt.de \\ ${ }^{3}$ Deutsches Kunststoff-Institut, Schloßgartenstrasse 6, 64289 Darmstadt, 06151-162405, \\ dlellinger@dki.tu-darmstadt.de \\ ${ }^{4}$ Deutsches Kunststoff-Institut, Schloßgartenstrasse 6, 64289 Darmstadt, 06151-162404, \\ ialig@dki.tu-darmstadt.de
}

Bei der Entwicklung von Lacken und Klebstoffen sind zum Beispiel deren Trocknungs- bzw. Härtungsverhalten und Wasseraufnahme von entscheidender Bedeutung. Zur Evaluierung dieser Eigenschaften wurden in den letzten Jahren Ultraschallmessverfahren erforscht und entwickelt. Eine hier untersuchte Möglichkeit des Aufbaus der Ultraschallwandler besteht im Einsatz piezoelektrischer Polymere als Sensor- und Aktormaterial mit der Zielstellung, die Frequenzbereiche der Ultraschallwandler besser an die Messaufgabe bei der Lack- und Klebstoffuntersuchung anzupassen. Der Aufbau von Ultraschallmessköpfen auf Basis von piezoelektrischen PVDF-Copolymeren wird im Detail diskutiert und die Nutzung der entwickelten Messköpfe sowie der Ultraschallreflexionsmethode zur Untersuchung des Trocknungsverhaltens verschiedener Beschichtungen demonstriert.

\section{Einleitung}

Ultraschallwandler haben vielfältige Einsatzfelder in verschiedensten technischen Bereichen. Beispiele sind die Nutzung in medizinischen Geräten für die Untersuchung von Gewebestrukturen, in Systemen für die zerstörungsfreie Werkstoffprüfung oder der Einsatz zur Abstandsmessung. Eine relative neue Anwendung ist der Einsatz von Ultraschallwandlern zur Untersuchung des Trockungs- bzw. Härtungsverhaltens von Materialien wie z.B. Lacken und Klebstoffen, die typischerweise im flüssigen Zustand appliziert werden. Auf Grund der teilweise vorliegenden flüssigen Phase der Materialsysteme eignen sich insbesondere zerstörungsfreie Messverfahren für die Aufnahme wichtiger Kenngrößen wie Schubmodul und Longitudinalwellenmodul oder charakteristischer Zeitpunkte der Trocknung bzw. Härtung. Dazu wurde eine sogenannte Ultraschallreflexionsmethode [1,2] entwickelt und in mehreren Iterationsstufen optimiert. Erfolgreich wurde diese Methode schon für das Screening von Lacken eingesetzt $[3,4]$. Typischerweise bestehen derartige Systeme aus piezoelektrischen Wandlerschichten und einer akustischen Verzögerungsstrecke, z.B. einem Glaskörper. Auf der freien Fläche des Glaskörpers wird das zu untersuchende Material aufgestrichen. Die piezoelektrische Wandlerschicht ist auf der der Probe gegenüberliegenden Seite der Verzögerungsschicht aufgebracht und für die Nutzung im Aktor- und Sensormodus elektrisch kontaktiert. Basierend auf dem aktorischen piezoelektrischen Effekt wird durch die Dickenänderung des Wandlers eine Schallwelle erzeugt und in die Glasverzögerungsstrecke eingekoppelt. Nach Reflexion der Schallwelle an der Probe enthält diese Informationen über die mechanischen Eigenschaften der Probe. Basierend auf dem sensorischen piezoelektrischen Effekt wird die reflektierte Schallwelle detektiert und anschließend ausgewertet. Bisher kamen in den Ultraschallmessköpfen in der Regel piezoelektrische Keramiken oder piezoelektrische Oxide als funktionale Wandlerschichten zum Einsatz [5]. Die so aufgebauten Ultraschallwandler sind auf bestimmte Bereiche (kleiner $10 \mathrm{bzw}$. größer $50 \mathrm{MHz}$ ) hinsichtlich ihrer nutzbaren Resonanzfrequenzen $\left(f_{R}\right)$ festgelegt. Neben den klassischen Piezoelektrika spielen zunehmend auch andere Materialien, wie Oxide und Polymere eine Rolle im Bereich der Ultraschallsensorik und -aktorik. Gerade der Einsatz von Polymeren als piezoelektrische Wandler gestattet einerseits das Einstellen der relevanten akustischen Eigenschaften über die Anpassung der Resonanzfrequenz in dem gewünschten Bereich sowie hat weitere Vorteile bei der Präparation der Wandler auf den Glasverzögerungsstrecken und bei der Strukturierung der Wandlerschichten. Die technologischen Herausforderungen bestehen dabei insbesondere in der Systemintegration der piezoelektrischen Wandlermodule auf der Glasverzögerungsstrecke, ihrer Strukturierung und Kontaktierung.

Hier stellen wir derartige Ultraschallwandlern mit Polymeren als funktionale, piezoelektrische Schichten mit Resonanzfrequenzen im Bereich von $10 \mathrm{MHz}<f_{R}<50 \mathrm{MHz}$ vor. Im Detail wird die Präparation der 
Wandler, deren Funktionalisierung und die Charakterisierung ihrer Ultraschalleigenschaften diskutiert. Zudem wird die Integration der Wandler, sowie der experimentelle Aufbau zur Analyse der Ultraschalleigenschaften dargestellt. Zur Demonstration der Anwendbarkeit der polymeren piezoelektrischen Wandler in der Ultraschallreflexionsmethode werden Untersuchungen zum Härtungsverhaltens eines Epoxidharzes, eines Polyurethans sowie einer Acrylatbeschichtung diskutiert. Aufbauend auf den Präparationseigenschaften polymerer Wandler, die einen strukturierten Polymerauftrag auf den Verzögerungsstrecken erlauben, werden zudem die Präparation und der Einsatz von Ultraschallwandlern in verschiedenen Array-Strukturen dargestellt. Dazu zählen Systeme, die auf einer Verzögerungsstrecke bis zu 16 gleichartige Wandler aufweisen.

\section{Ultraschallverfahren}

Die hier eingesetzten Ultraschallsensoren bestehen aus der funktionalen piezoelektrischen Wandlerschicht und einer akustischen Verzögerungsstrecke, einem Glaskörper (Fa. Schott, Typ F7). Auf der freien Fläche des Glaskörpers wird das zu untersuchende Material (Probe) aufgestrichen. Die piezoelektrische Wandlerschicht ist typischerweise ein dünner Film bzw. eine dünne Schicht, die auf der der Probe gegenüberliegenden Seite der Verzögerungsstrecke aufgebracht ist. Die piezoelektrischen Schichten werden mittels aufgedampfter Elektroden elektrisch kontaktiert und durch einen Impulsgenerator angesteuert. Basierend auf dem aktorischen piezoelektrischen Effekt wird durch die Dickenänderung des Wandlers eine Schallwelle erzeugt und in die Glasverzögerungsstrecke eingekoppelt. Diese Schallwelle trifft auf die Probe und enthält nach der Reflexion an dieser die Informationen über deren mechanische Eigenschaften. Die reflektierte Schallwelle wird durch den Ultraschallwandler im Sensormodus aufgenommen, das Signal verstärkt und der Auswerteelektronik und -software zugeleitet (Abb. 1 (a)). Dieses Grundprinzip der Messung ist in verschiedenen Arbeiten beschrieben [1-4]. Die Glasverzögerungsstrecke mit dem piezoelektrischen Wandler ist in Abbildung 1 schematisch (Abb. 1 (b)) sowie als Foto (Abb. 1 (c)) für einen einkanaligen Sensor dargestellt. Die den Glaszylinder aufnehmende Messzelle ist ausführlich in Referenz [6] beschrieben.
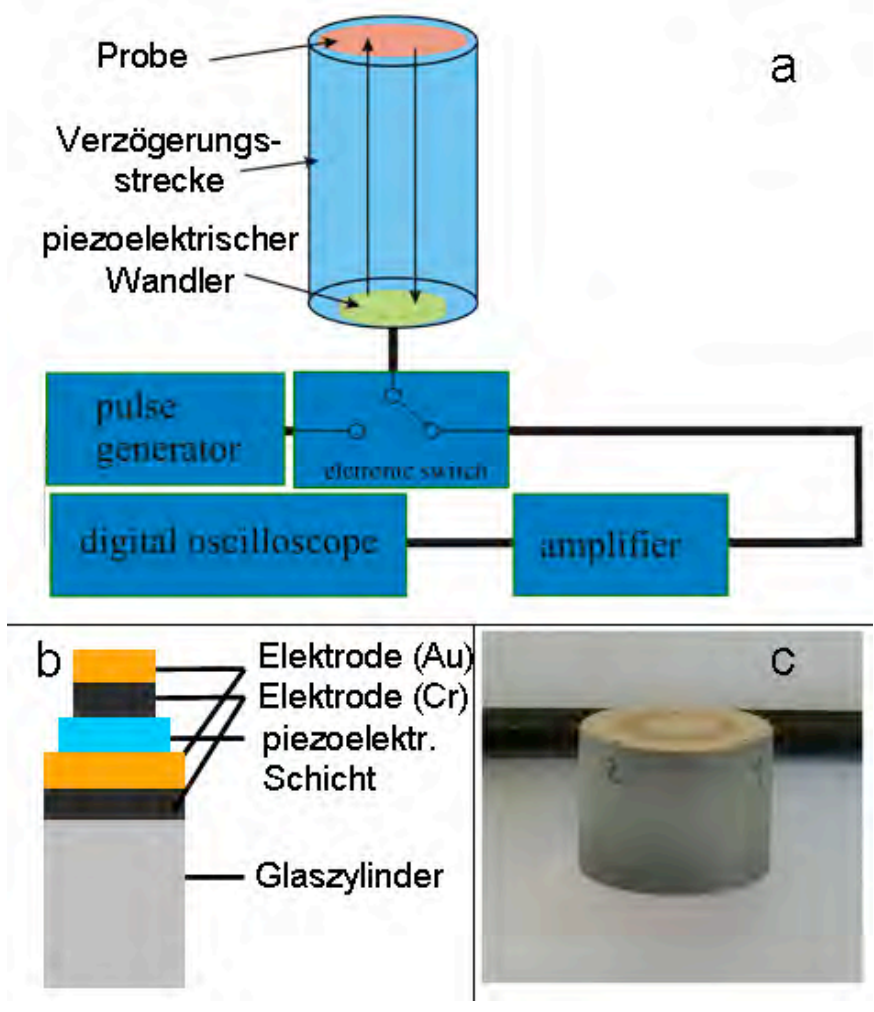

Abb. 1: Schema des Messaufbaus der Ultraschallreflexionsmethode (a) sowie des auf der Verzögerungsstrecke präparierten polymeren Wandlers (b) in Seitenansicht.

Foto des Polymerwandlers auf der Glasverzögerungsstrecke (c). 
Nach dem gleichen Prinzip wurde eine 16-kanalige Ultraschallmesszelle aufgebaut. Dabei kam ein Multiplexer zur Signalverteilung zum Einsatz. Schematisch ist der Aufbau in Abbildung 2 gezeigt. Dazu wurden 16 Wandler auf einem flächigen Glassubstrat aufgebaut, wobei jeweils 8 Wandler in einer Reihe angeordnet wurden. Abbildung 2 zeigt neben der schematischen Darstellung wiederum ein Foto eines 16-kanaligen Sensors auf Basis einer piezoelektrischen Polymerschicht.
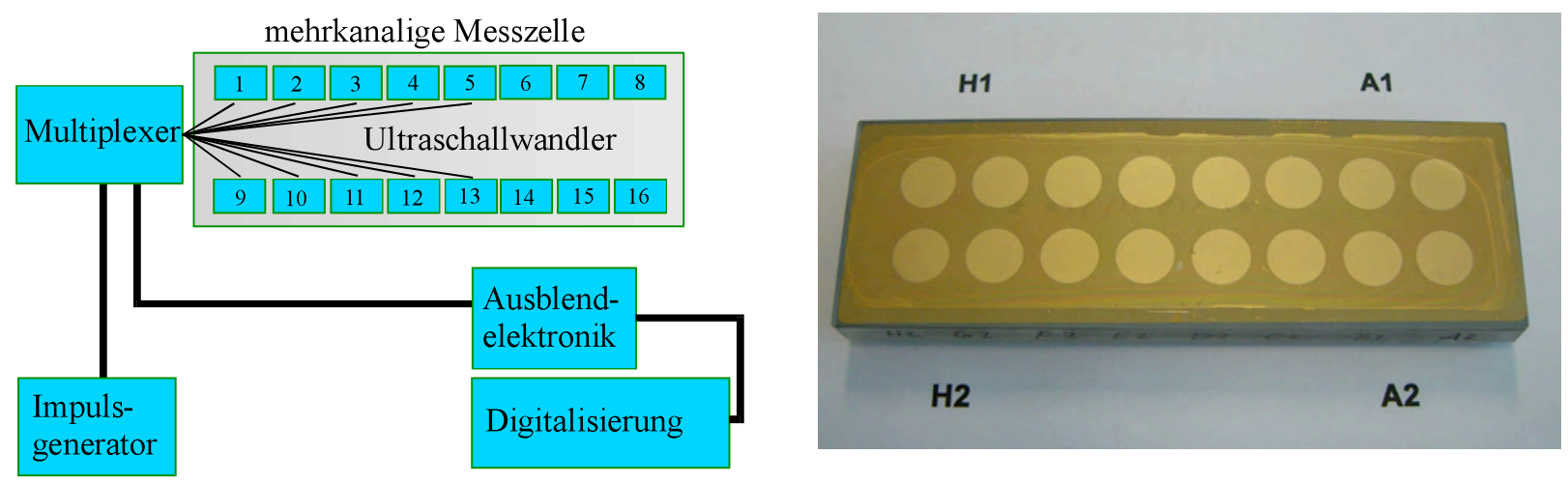

Abb. 2: Aufbau des Messplatzes für mehrkanalige Ultraschallsensoren (links) sowie Foto eines 16-kanaligen Ultraschallsensors aufgebracht auf einer flächigen Glasverzögerungsstrecke mit der Dimension $114 \mathrm{~mm} \times 34 \mathrm{~mm}$ (rechts).
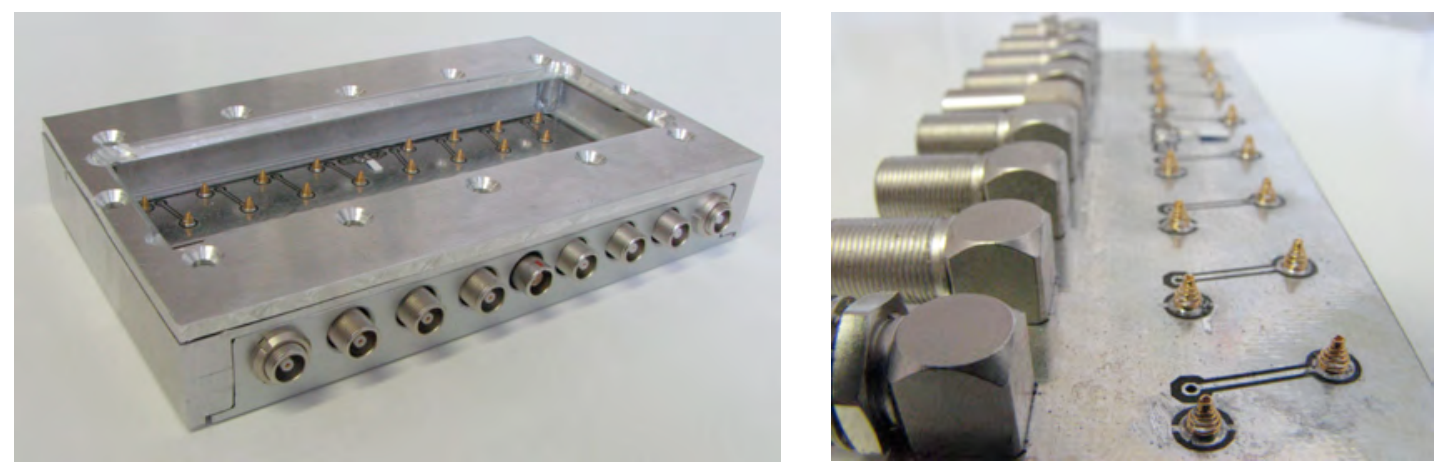

Abb. 3: Messzellengehäuse für den 16-kanaligen Sensor (links) sowie vergrößerte Ansicht einer Platine zur Kontaktierung von 8 Wandlern (rechts).

Die entwickelte Messzelle zur Aufnahme des 16-kanaligen Ultraschallsensors ist in Abbildung 3 gezeigt. Eine der unten in der Messzelle integrierten Kontaktierungsleisten für 8 Einzelwandler ist im rechten Bild der Abbildung dargestellt. Nach Integration der Ultraschallsensoren in die Messzelle erfolgten Messungen in einem Klimaschrank zur Evaluierung der piezoelektrischen Wandler sowie auch zum Screening von auf der freien Glasfläche aufgebrachten Lacksystemen.

\section{Präparation der Polymerschichten}

Als piezoelektrisches Polymer wurde Poly(vinylidenfluorid-Trifluorethylen) (P(VDF-TrFE)) ausgewählt und prozessiert. Dieses polare, teilkristalline Copolymer des Polyvinylidenfluorids (PVDF) liegt nach der Extrusion bzw. dem Aufbringen aus der flüssigen Phase bereits in einer polaren, ferroelektrischen Modifikation vor [7]. Die Ferroelektrizität beruht dabei auf der Orientierung und Stabilisierung der Ausrichtung molekularer Dipole in der Polymerkette. Die Aufbringung aus dem flüssigen Zustand hat folgende Vorteile: Zum einen sind keine akustischen Koppelschichten zwischen Polymerwandler und Glassubstrat notwendig und andererseits sind praxistaugliche Applikationsverfahren (Drucken, Sprühen, etc.) zur Wandleraufbringung anwendbar. So können beliebige Wandlergeometrien realisiert werden.

Vorversuche erfolgten einerseits zur Evaluierung der Haltbarkeit der Elektrode und zur Optimierung des aus verschiedenen Materialien geschichteten Elektrodenaufbaus auf den Glasverzögerungsstrecken sowie andererseits zum Aufbringen der Polymerschicht mittels verschiedener Verfahren wie 
Aufschleudern (spin coating), Gießen (drop casting) und Aufsprühen (air-brush technology). Auf Grund dieser Entwicklungsarbeit sowie insbesondere hinsichtlich der Reproduzierbarkeit ihrer Schichtdicke (und somit der Resonanzfrequenz) wurden die in Abbildung 1 und 2 gezeigten und in Abbildung 4 bis 7 charakterisierten Polymerwandler durch eine Gießtechnik in Kombination mit verschiedenen Temperregimen zum Verdampfen der Lösungsmittel aufgebracht.

\section{Polarisierung der Polymerschichten}

Nach der Metallisierung der zuvor beschriebenen nicht strukturierten und strukturierten Elektroden erfolgte die elektrische Polarisierung. Hierfür wurde ein Verfahren entwickelt, welches die Messung des Polungsstromes durch die Probe bei der bipolaren Variation des über der Probendicke anliegenden elektrischen Feldes und somit die Berechnung der Polarisation in der Probe gestattet [8]. Die mit dem Verfahren gewonnenen Ergebnisse sind exemplarisch für den Einzelwandler mit der Bezeichnung A2 des in Abbildung 2 (rechts) gezeigten 16-kanaligen Sensors in Abbildung 4 dargestellt. Hier zeigt sich, dass die aus der Lösung prozessierten P(VDF-TrFE)-Wandler bis zur Sättigung ihrer Polarisation elektrisch polarisiert werden konnten, wobei die erreichten Polarisationswerte denen herkömmlicher P(VDF-TrFE)Wandlerfolien entsprechen. Eine vergleichbare Polarisation wurde auch bei den anderen Einzelwandlern erreicht. Trotz umfangreicher Untersuchungen zum Aufbringen homogener Schichten und Vermeidung von Randüberhöhungen traten teilweise Schwierigkeiten bei der Polarisierung einzelner Wandler im Randbereich des 16-kanaligen Ultraschallsensors auf, die in Schichtdickenschwankungen und strukturellen Änderungen begründet sind.

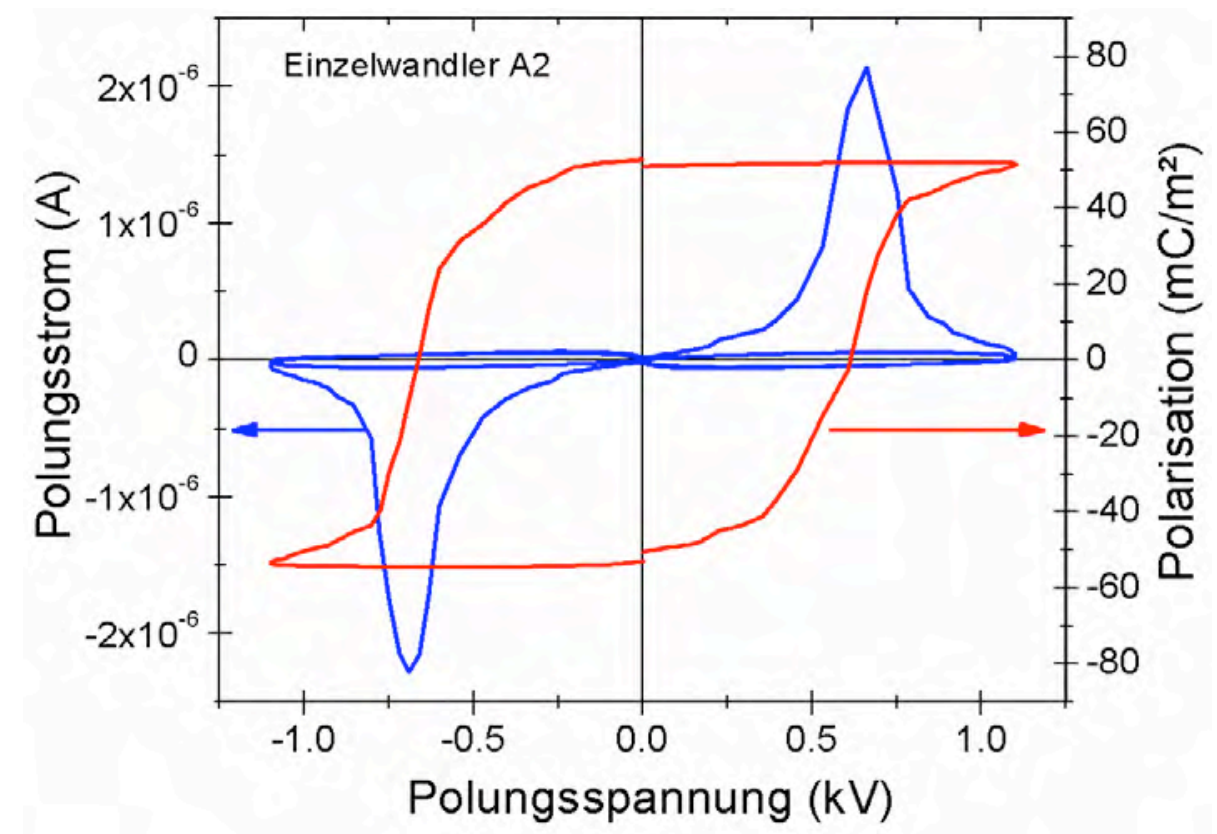

Abb. 4: Am Einzelwandler A2 des in Abbildung 2 gezeigten 16-kanaligen Sensors gemessener Polungsstrom sowie die daraus berechnete Hysterese der Polarisation in Abhängigkeit vom elektrischen Feld.

\section{Charakterisierung der Polymerwandler}

Für die Einzelwandlern $\mathrm{A} 1$ und $\mathrm{H} 1$ des hier beschriebenen 16-kanaligen Ultraschallsensors sind die geringeren Polarisationswerte auch an Hand der gemessenen piezoelektrischen Koeffizienten im Sensormodus in Abbildung 5 dargestellt. Alle anderen Einzelwandler zeigen die auf Grund des verwendeten Polymers erwartete piezoelektrische Aktivität im Bereich von etwa 10 bis $13 \mathrm{pC} / \mathrm{N}$. Die Einzelwandler des 16-kanaligen Sensors zeigen je nach Präparation Schichtdicken zwischen 15 und ca. $30 \mu \mathrm{m}$, was Resonanzfrequenzen für die Dickenschwingung der Wandler im Bereich von 18 bis $26 \mathrm{MHz}$ bedingt. Für den in Abbildung 2 gezeigten 16-kanaligen Sensor sind die entsprechenden Schichtdicken und Resonanzfrequenzen in den Abbildungen 6 und 7 (links) zusammengestellt. Die Bandbreite der Wandler (Dämpfung um $6 \mathrm{~dB}$ ) beträgt $9.5 \pm 0.5 \mathrm{MHz}$. Dadurch lässt sich bei einer mittleren 
Anregungsfrequenz der Wandler von $22 \pm 4 \mathrm{MHz}$ eine ausreichende Signalamplitude erzeugen. Die Amplituden liegen (ohne den zuvor diskutierten abweichenden Wandler A1) zwischen 0,8 und 2,3 V gemessen bei einer Anregungsspannung von $5 \mathrm{~V}$ und einer Verstärkung von $25 \mathrm{~dB}$, vergleiche Abbildung 7 (rechts).

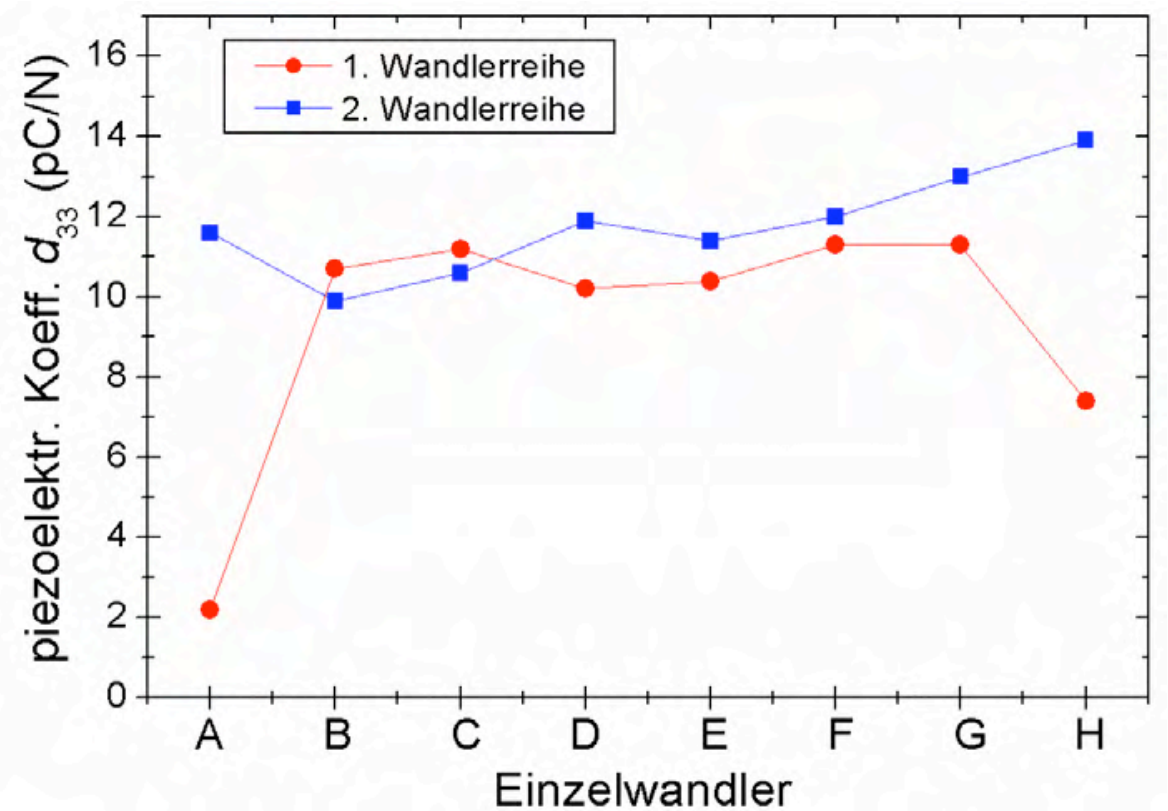

Abb. 5: Im Sensormodus gemessene piezoelektrische Aktivität der Einzelwandler des in Abbildung 2 gezeigten 16-kanaligen Wandlers.

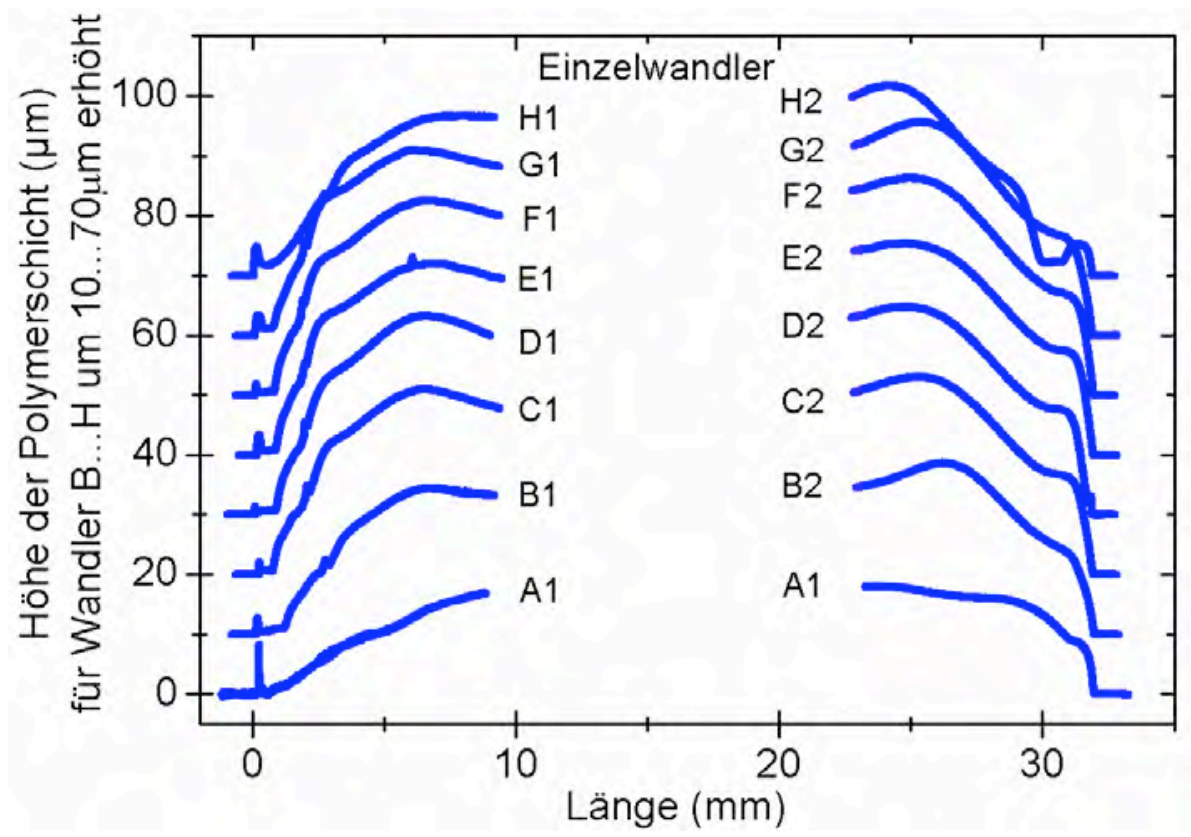

Abb. 6: Dickenprofile der einzelnen P(VDF-TrFE)-Wandlerschichten des in Abbildung 2 gezeigten 16kanaligen Wandlers. Zur besseren Lesbarkeit sind die Profile der Einzelwandler B bis H jeweils um $10 \mu \mathrm{m}$ erhöht zum vorhergehenden Wandler dargestellt.

\section{Einsatz der Ultraschallwandler zum Screening von Lacken}

Die mit dem beschriebenen Ultraschallsensor entwickelte Ultraschallmesszelle ist für die Untersuchung dünner Polymerschichten mit Schichtdicken ab etwa $20 \mu \mathrm{m}$ geeignet. Der Longitudinalwellenmodul von bis zu 16 unterschiedlichen Polymerfilmen lässt sich zeitaufgelöst während der Härtung oder Trocknung bestimmen. Abb. 8 zeigt exemplarisch die Härtungs- bzw. Trocknungsverläufe dreier typischer 
Polymersysteme (Acrylat-Dispersion, Polyurethan und Epoxidharz) im Vergleich. Die charakteristisch unterschiedlichen Verfestigungskinetiken lassen sich klar unterscheiden. Der Übergang von einer wässrigen Dispersion zu einem geschlossenen Film verläuft erwartungsgemäß schnell im Vergleich zur Härtung des Epoxidharzes und des Polyurethans. Für das Epoxidharz und das Polyurethan sind aufgrund der für diese Stoffklassen geringen Härtungstemperatur sehr langsame Kinetiken zu erwarten. Auch die Modulwerte der ausgehärteten Proben unterscheiden sich gemäß ihrer unterschiedlichen Glasübergangstemperaturen. Das Epoxidharz mit einer Glasübergangstemperatur von $55^{\circ} \mathrm{C}$ hat mit etwa $8 \mathrm{GPa}$ einen wesentlich größeren Modul als das Polyurethan und der Acrylatfilm mit Glasübergangstemperaturen von $6^{\circ} \mathrm{C}$ bzw. $5^{\circ} \mathrm{C}$. $8 \mathrm{GPa}$ entsprechen dabei einem Modulwert eines verglasten Polymers, $4 \mathrm{GPa}$ eher eines Polymers im Bereich des Glasübergangs, indem sich das Polyurethan und der Acrylatfilm aufgrund der Messfrequenz von $22 \mathrm{MHz}$ bei der Messtemperatur von $23^{\circ} \mathrm{C}$ befinden (Temperatur-Frequenz-Superpositionsprinzip) $[9,10]$. Analog ist diese Ultraschallmessmethode auch zur Untersuchung der Trocknung oder Härtung anderer Produktklassen, z. B. Klebstoffe, geeignet.
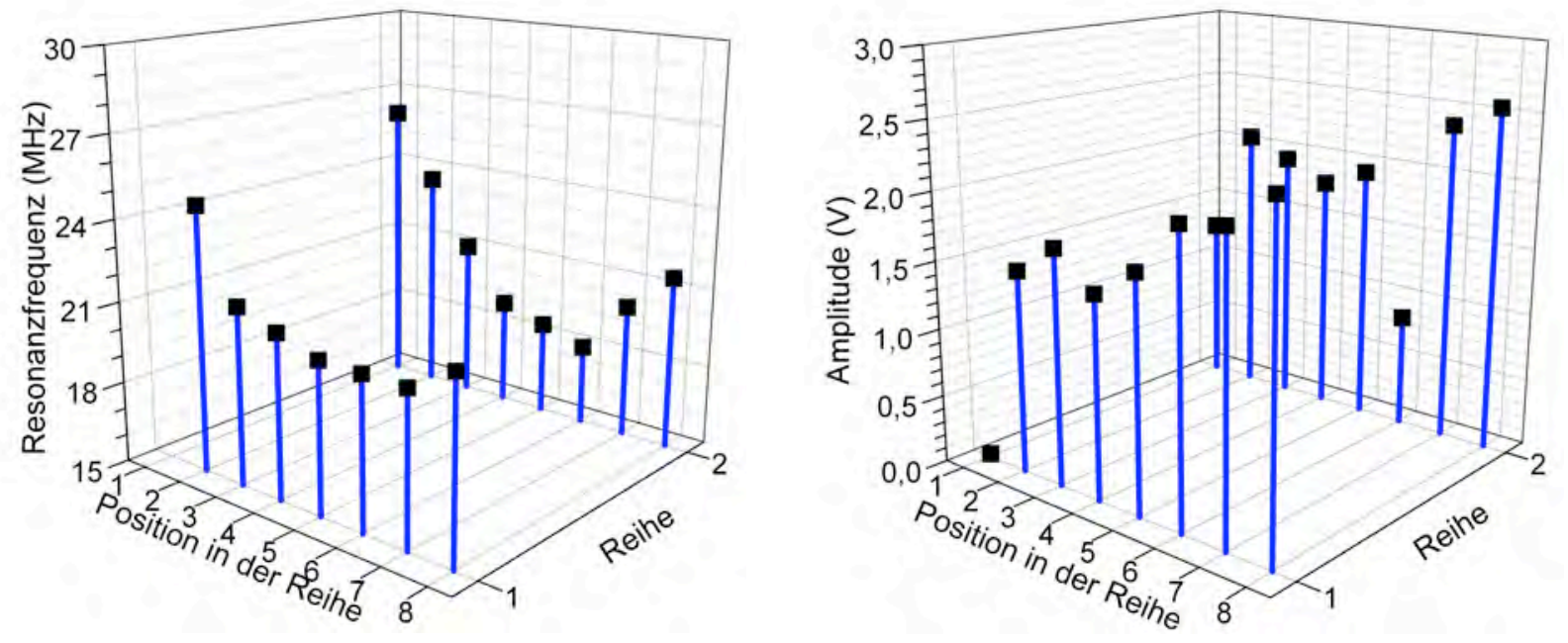

Abb. 7: Resonanzfrequenzen (links) und Amplituden (rechts) der einzelnen P(VDF-TrFE)Wandlerschichten des in Abbildung 2 gezeigten 16-kanaligen Wandlers.

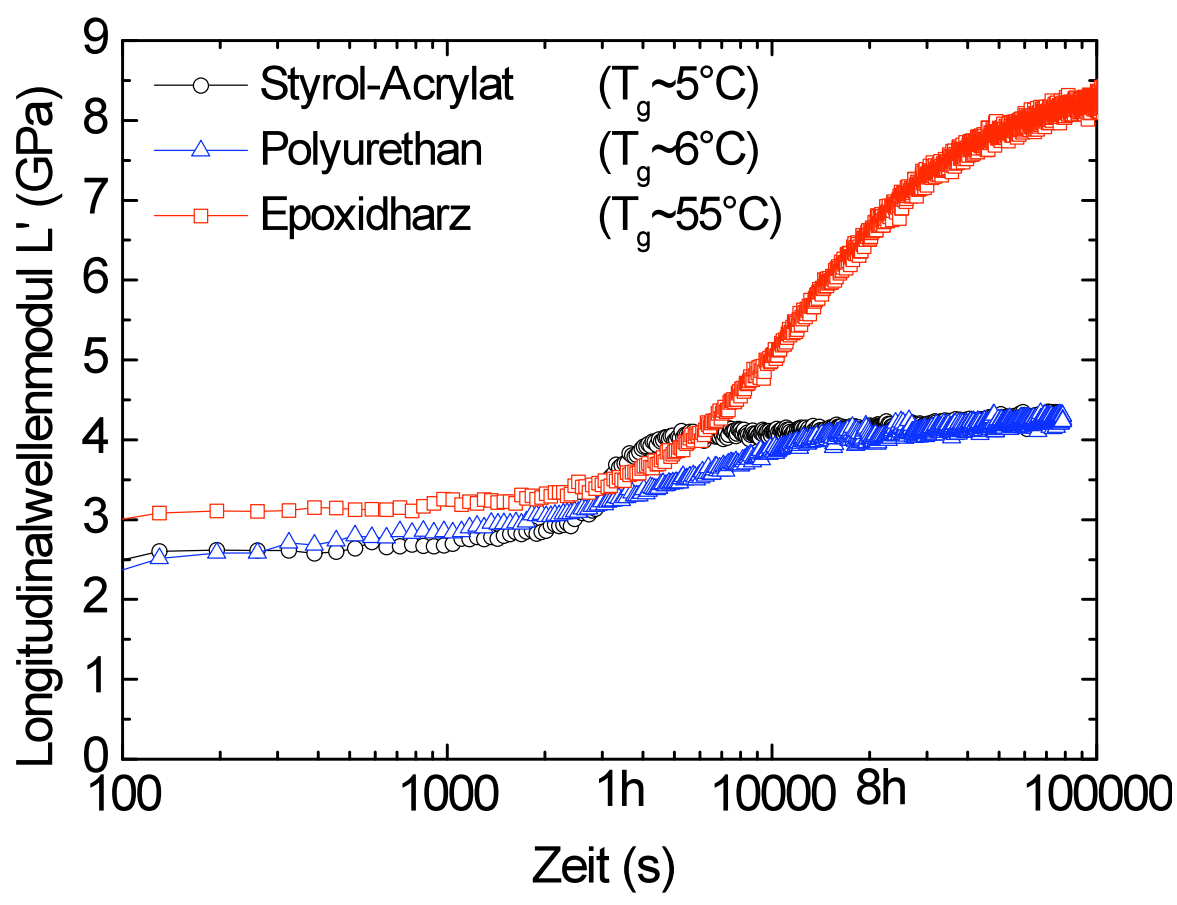

Abb. 8: Vergleich der Härtung bzw. Trocknung von Epoxidharz-, Polyurethan- und Acrylatbeschichtungen. 


\section{Zusammenfassung}

Die Nutzung der Ultraschallreflexionsmethode mit piezoelektrischen Polymerwandlern wurde erfolgreich zur Untersuchung der mechanischen Eigenschaften verschiedener Beschichtungen während der Trocknung bzw. Härtung demonstriert. Unter Nutzung des ferroelektrischen Polymers P(VDF-TrFE) erfolgte der Aufbau von Ultraschallwandlern mit verschiedenen im Frequenzbereich von 10 bis $50 \mathrm{MHz}$ liegenden Resonanzen. Exemplarisch wurden hier das elektrische Polungsverhalten sowie die piezoelektrische Aktivität eines Systems mit Resonanzfrequenzen von ca. $22 \mathrm{MHz}$ dargestellt. Die Ergebnisse weisen ein dipolares Schalten als Grundlage der Polarisation sowie eine Sättigung im Polarisationsaufbau nach. Basierend auf dieser ferroelektrischen Polarisation zeigen die P(VDF-TrFE)Wandler piezoelektrische Aktivitäten mit piezoelektrischen Koeffizienten größer als $10 \mathrm{pC} / \mathrm{N}$. Sowohl mit den entwickelten 1-kanaligen wie auch mit den entwickelten 16-kanaligen Ultraschallmesszellen konnte die Härtung bzw. Trocknung von Epoxidharz-, Polyurethan- und Styrol-Acrylat-Beschichtungen vermessen sowie die unterschiedlichen Kinetiken der Verfestigungsprozesse nachgewiesen werden.

\section{Danksagung}

Die Autoren danken für die Förderung des IGF-Vorhabens $14953 \mathrm{~B}$ der Forschungsvereinigung Forschungsgesellschaft Kunststoffe e. V., Schlossgartenstraße 6, 64289 Darmstadt zum Thema „Ultraschallsensoren mit ferroelektrischen Polymerschichten für das Screening von Lacken und Klebstoffen" über die AiF im Rahmen des Programms zur Förderung der industriellen Gemeinschaftsforschung und -entwicklung (IGF) vom Bundesministerium für Wirtschaft und Technologie. Die Mitarbeiter des Fraunhofer IAP danken zudem dem Bundesministerium für Bildung und Forschung (BMBF) für die Förderung ihres Arbeitsschwerpunktes „piezoelektrische Funktionsschichten“ im Rahmen des nanett-Projektes (Forschungsvorhaben 03IS2011D: Spitzenforschung in den Neuen Ländern Kompetenznetzwerk für Nanosystemintegration, Teilprojekt: Materialintegrierte Sensorik).

\section{Literatur}

[1] I. Alig, D. Lellinger, J. Sulima, S. Tadjbakhsch, Filmbildung von Lacken und Klebstoffen, Farbe \& Lack 102, 56-62 (1996).

[2] I. Alig, D. Lellinger, J. Sulimma, and S. Tadjbakhsch, Ultrasonic shear wave reflection method for measurements of the viscoelastic properties of polymer films, Review of Scientific Instruments 68, 1536-1542 (1997).

[3] I. Alig, S. Tadjbach, D. Lellinger, H. Oehler, M. Wenzel, Auf Hertz und Nieren: Ultraschallverfahren zur Verfolgung von Trocknung und Verfestigung von Lacken und Klebstoffen, Farbe \& Lack 109, 76-79 (2003).

[4] H. Oehler, D. Lellinger, I. Alig, Filmbildung von Dispersionen mit Ultraschall verfolgen, Farbe \& Lack 111 (7), 22-24 (2005).

[5] I. Alig, H. Oehler, D. Lellinger, and S. Tadjbach, Monitoring of film formation, curing and ageing of coatings by an ultrasonic reflection method, Progress in Organic Coatings 58, 200-208 (2009).

[6] M. Wegener, H. Oehler, D. Lellinger, and I. Alig, Piezoelectric polymers as transducers for the ultrasonic-reflection method and the application in mechanical property-screening of coatings, Review of Scientific Instruments 83, 016102 (2012).

[7] H.S. Nalwa (Ed.), Ferroelectric Polymers, Marcel Dekker Inc. (1995).

[8] M. Wegener, Polarization - electric field hysteresis of ferroelectric PVDF films: Comparison of different measurement regimes, Review of Scientific Instruments 79, 106103 (2008).

[9] G. Strobl, The Physics of Polymers, Springer (1997).

[10]E. Donth, The Glass Transition, Springer (2001). 